\title{
Applying the Master Narrative Framework to Gender Identity Development in Emerging
}

\section{Adulthood}

\author{
Kate C. McLean ${ }^{1}$ \\ Hannah Shucard ${ }^{1}$ \\ Moin Syed ${ }^{2}$ \\ ${ }^{1}$ Western Washington University \\ ${ }^{2}$ University of Minnesota
}

McLean, K. C., Shucard, H., \& Syed, M. (2017). Applying the Master Narrative Framework to Gender Identity in Emerging Adulthood: Emerging Adulthood, 5, 93-105.

Key words: Identity development, emerging adulthood, master narratives, gender identity

Author Note. We thank Kari Vanderburg, Colton Dion, Vinitha Vithayathil, Haley Miller-Boren, Lynell Solene, Jaynee Bohart, and Alisha Yoder for coding and data collection, and our participants for their time. Correspondence can be addressed to the first author at: Western Washington University, 516 High Street, MS 9172, Bellingham, WA 98225;

Kate.McLean@wwu.edu

Key words: Identity development, emerging adulthood, master narratives, gender identity 


\begin{abstract}
We applied a novel framework to identity development, capturing both content and process, and personal and cultural components of identity in the content domain of gender. We examined master (and alternative) narratives: ubiquitous, powerful cultural stories with which individuals negotiate in constructing personal identity. Study 1 was a quantitative investigation of personal narratives about gender $(n=245)$; Study 2 was a qualitative study of discourse processes concerning gender roles $(n=12)$. Employing three approaches to identity development (status, narrative, and discursive) in a mixed methods-design, we found that most individuals position themselves around a newer, alternative narrative about gender equality. However, in social interactions this narrative got less airtime, in favor of narratives representing either traditional views, or gender equality. Further, those who were exploring their identities were more likely to employ the less traditional narrative, suggesting a link between changing master narratives and personal identity exploration.
\end{abstract}




\section{Applying the Master Narrative Framework to Gender Identity Development in Emerging Adulthood}

Identity development is the major psychosocial task of adolescence and emerging adulthood (Arnett, 2000; Erikson, 1968; McLean \& Syed, 2015a). However, our current understanding of identity development is limited by at least two issues: insufficient attention to the content of identity development (McLean, Syed, \& Shucard, 2016; McLean, Syed, Yoder, \& Greenhoot, 2014; Syed \& Azmitia, 2010), and insufficient inattention to how personal and cultural aspects of identity intersect (Hammack, 2011; McLean \& Syed, 2016; Way \& Rogers, 2015). An examination of process tells us how individuals are developing, whereas an examination of content tells us what is developing. When examining both process and content it becomes clear that identity contents are culturally defined, and that different processes are required for managing different contents. Thus, attention to both personal and cultural components, and the processes and contents, of identity development are necessary for a full understanding of the phenomenon.

In the present studies our aim was to apply a recent framework (McLean \& Syed, 2016) that captures the intersection of personal identity narratives and cultural master narratives within one specific content domain: gender identity. This framework captures the processes of negotiation with and internalization of larger cultural structures, which in this case were conceptions of and beliefs about gender. In applying this framework, we took an integrative approach by employing what have been relatively divergent perspectives to the study of identity development, each with differing emphases on both content and process, and personal and cultural aspects of identity: narrative, identity status, and discursive approaches.

\section{Three Approaches to the Study of Identity Development}


Erikson (1968) proposed that a major psychosocial task that takes center stage during adolescence and emerging adulthood is that of defining oneself. To Erikson, this meant constructing a sense of felt coherence across time, context, and within one's culture (see also Syed \& McLean, in press). As we detail below, the two most common contemporary approaches to identity development have emphasized the processes of identity development, with less empirical effort attending to the content, or object, of those processes. Both of these approaches have also emphasized the personal aspects of identity development, with less attention to the cultural context of identity development (cf., Hammack, 2008). This is a notable gap because Erikson (1968) conceptualized identity development as a process of re-negotiating childhood identifications as one becomes aware of the structures in which one is living, and how to live within those structures. However, the third approach (i.e., discursive) has examined culture more clearly, through focusing more on the enactment of identities at particular moments, rather than the personal continuity emphasized by Erikson. The framework we provide captures these aspects of Erikson's original theory, and we employ each approach in our methodology for a fully integrative examination of identity development. We apply our framework to the content domain gender identity, which has clear relevance to intersections of personal and cultural experiences. We briefly review each of these approaches below, and then present our integrative framework.

The Identity Status Approach. The identity status literature captures the personal processes of identity exploration and commitment, which are predominantly assessed via survey measures (Crocetti \& Meeus, 2015; Kunnen \& Metz, 2015). Identity content, and thus cultural context and structures, has been relatively overlooked in this work. Exceptions include research driven by interview assessments, which allow contents to be more fully elaborated by 
participants, and which shows that cultural structures are reflected in personal identities (Kunnen \& Metz, 2015). For example, Archer (1989) found that adolescent females engaged in more identity exploration in the domain of family roles than did males (see also Frisén \& Wängqvist, 2011).

Narrative Approaches. Narrative approaches focus on the construction of a personal life story, a selective and subjective reconstruction of the personal past that serves to define the self (McAdams \& McLean, 2013). Like the status literature, the content of gender has been relatively neglected here, though the examination of sex differences in narrative construction is more prevalent. For example, in constructing personal stories females are more elaborative than are males (e.g., Pasupathi \& Wainryb, 2010; Zaman \& Fivush, 2011; see Grysman \& Hudson, 2013 for a review). However, researchers have recently argued that it is not being male or female that matters for narrative construction, but how one identifies oneself in relation to cultural norms and expectations about being male or female, or how gender-typical one is (e.g., Grysman \& Hudson, 2013; Grysman, Merrill, \& Fivush, 2016).

Discursive Approaches. Similar to the narrative perspective, discursive approaches focus not on sex differences, but on how gender is constructed (e.g., Bamberg, 2004; Korobov \& Bamberg, 2004). However, unlike narrative and status approaches to identity, internal and stable aspects of self are not assumed (see Thorne, 2004). Instead, researchers examine how identities are enacted in discourse as individuals 'position' themselves in relation to each other and to master narratives; positioning is a way to examine the 'doing,' or performing, of identity (Korobov, 2010). In a study examining how adolescent boys construct masculinity, Korobov and Bamberg (2004) argued that flexibly positioning oneself around gendered master narratives reflected mature developmental processes. This approach is in particular contrast to survey 
measures that do not allow for this kind of dynamic observation of culturally valued and mediated processes (Korobov \& Bamberg, 2004). In study 2 we employ this perspective, but note that we do not adopt all components of it, as we see these master narrative positions as leading towards internalization (McLean \& Syed, 2016), rather than as relatively fleeting 'performances.'

\section{The Master Narrative Framework for Identity Development}

In examining the contemporary literature, and its divergent approaches, we have recently argued that master narratives provide a framework for examining the personal and the cultural, and process and content, as they are all relevant to identity development (McLean \& Syed, 2016; see also McLean, 2015; Syed \& McLean, in press). Master narratives are culturally shared stories that provide guidance for how to belong to, and be a good member of, a given culture; they are useful frameworks that guide personal story construction. Two processes link personal and master narratives: individuals negotiate with master narratives in constructing their own stories, which leads to the internalization of master narratives as part of one's identity. In other words, using a master narrative framework allows us to understand personal identity development as a process of "narrative engagement" (Hammack \& Cohler, 2009), in which individuals negotiate with and internalize master narratives as parts of their identities.

Yet, these identity processes are not entirely governed by the individual (McLean \& Syed, 2016). There is a power to master narratives stemming from their historical weight and ubiquitous use, which motivates community members to fit in to earn acceptance from others. Indeed, these narratives are relatively rigid and can thus constrain the individual, which is made particularly apparent when the master narrative does not provide a framework that aligns with one's own experience. This makes the negotiation process quite conscious, and challenging, as 
individuals seek alternative narratives to guide personal story construction. As we articulate below, gender is a prime identity content to which we can apply this framework within the U.S. because of the long-standing differential power conferred to males and females, paired with ongoing discourse and social policies that emphasize gender equality. Thus, in contemporary U.S. society, there is clearly potential room for negotiation with cultural expectations about gender and identity.

\section{Gender and Development}

Traditionally, gender identity has been defined as the ways in which people understand themselves as male or female in the cultural contexts in which they are developing (e.g., Wood \& Eagly, 2015). Precursors to these understandings begin early (see Leaper \& Friedman, 2007; Martin, Ruble, \& Szkrybalo, 2002 for reviews). By the first year infants can distinguish physical attributes that are gender-linked, such as faces. By two years of age toddlers employ gender labeling, and by three, they are aware of their own gender, known as gender identity.

Research on gender identity in adolescence and emerging adulthood is less common than

in childhood and has focused on gender typicality (e.g., Jewell \& Brown, 2014; Smith \& Leaper, 2006), rather than identity per se. That is, whereas identity development is usually conceptualized as engaging in some kind of reflective or exploratory process of understanding various aspects of oneself (including gender), the research on gender typicality generally assesses where one falls along the dimensions of femininity and masculinity and does not capture processes of identity work. This disconnect between these two areas of research is notable because this is the developmental stage when identity development becomes the main psychosocial task, and when awareness of larger cultural and societal structures - many of which contain messages about gender - emerges and becomes personally salient (Erikson, 1968; see 
also Flanagan, Kim, Pykett, Finlay, Gallay, \& Pancer, 2014; Thorne \& Nam, 2007). Further, although the identity development literature centers squarely on adolescence and emerging adulthood, somewhat ironically, researchers here have left gender relatively under-examined in comparison to other domains of identity, such as ethnic identity (e.g., Umaña-Taylor et al., 2014) or content domains such as religion or relationships (e.g., McLean et al., 2015; 2016). Thus, we situated this study in emerging adulthood, a time when individuals are exploring culturally salient adult roles such as family and work-related issues (see Mayseless \& Keren, 2014), which are tightly linked to gender norms (e.g., Archer, 1989; Frisén \& Wängqvist, 2011; Shmuel, Laursen, \& Dickson, 2014).

As we discussed above, although the domain of gender has been relatively underexamined in emerging adult identity research, all approaches to identity development share the idea that engagement with this domain is developmentally important. That is, identity exploration, narrative elaboration, and nuanced positioning are indicative of more mature identity development. All approaches also complement each other by differentially targeting cultural and personal processes; therefore, combining them creates an integrative framework for studying identity development.

We examined both personal processes related to the development of gender identity, and how those personal processes were linked to master narratives. In Study 1 we focused on processes of internalization by identifying master and alternative narratives in individuals' personal stories, and examined whether internalization of these different narratives was related to processes of personal identity development. In Study 2 we focused on negotiation by examining how personal stories are used to negotiate with master narratives about gender in a conversational context. We emphasize that our intention was to apply the master narrative 
framework to the content domain of gender identity. In making this application, however, we also presumed that we would learn something about gender identity, beyond the typical assessments of masculinity or femininity, particularly in terms of how individuals work within and with culture to understand gender as a component of their identities.

\section{The Present Studies}

We addressed the relation between personal, alternative, and master narratives in the context of one type of master narrative - the life course master narrative (McLean \& Syed, 2016). At a basic level many cultures have expectations about the ordering and timing of life events, such as graduation from school, getting a job, entering into a committed romantic relationship, and having children (Habermas, 2007; Rubin \& Bernsten, 2003). Although the plot of expected events may be the same for men and women, the emphasis differs. For example, in the U. S., where this research was conducted, although both men and women are expected to have children, women are expected to be more nurturing, and to put more time and effort into this life task, and in fact do so (e.g., Sayer, Bianchi, \& Robinson, 2004). This reflects a traditional master narrative, with which one may position oneself toward or against.

However, identifying master narratives is not so easy. In a prior study we examined identity contents in a variety of personally important identity narratives in samples of college students from three very different campuses (with data collected 10 years apart). We found no mention of the content of gender across 2214 narratives (in comparison to the other status content domains such as family, dating, and values) (McLean et al., 2014; see also Azmitia, Syed, \& Radmacher, 2008). That is, gender did not emerge as an explicit part of the content of their past experiences. We speculated that there may be a new narrative about gender equality that diminishes experiences in which gender is made salient. That is, people, may be either 
experiencing equality, or not attending to inequality to preserve an alternative to the master narrative - the equality narrative - in which the expectations for men and women are the same. That is, if men and women are now the same, the issue has become obsolete, and is not a part of the conversation. However, without directly asking about gender, this explanation remains untested.

In the present studies we explicitly addressed internalization of and negotiation with master narratives as identity processes. In Study 1 we examined what master and alternative narratives were internalized in telling personally important stories about gender. We also examined how narrative positions were related to identity processes. In Study 2 we examined how personal stories were used in negotiating with master and alternative narratives, particularly attending to processes that might influence the rigidity or flexibility of master and alternative narratives.

Beyond identifying master narrative positions, in Study 1 we also wanted to identify characteristics of the narratives that reflected each position, using several standard narrative coding systems relevant to identity processes. We coded the personal meaning made of the events as a measure of narrative identity development (e.g., McLean \& Pratt, 2006), as well as the affective valence of the events. Affect may be important because more negative, or disruptive, experiences are more likely to prompt identity processes such as exploration or meaning-making (e.g., McLean \& Thorne, 2003), and are also moments in which discursive processes reveal personal and cultural tensions (Korobov \& Bamberg, 2004). We also examined the degree to which participants experienced these events as reflecting a stable aspect of who they are versus a reflection of personal change. Although both are important to a sense of personal continuity (Pasupathi, Brubaker, \& Mansour, 2007), they reflect different ways of 
conceptualizing identity development and, critically, master narrative change may be related to these personal processes of change and stability (McLean \& Syed, 2016). Due to the lack of gender content in our previous study, we were also motivated to code the degree of narrative specificity, and whether the narrated event was one's own experience or someone else's. That is, if individuals do indeed have a difficult time recalling gender-related experiences, as our previous results suggest (McLean et al., 2014; McLean et al., 2016), determining the quality of these recollections in terms of how specific they were, as well as whether they were actual personal experiences, seemed warranted.

\section{Study 1: Method}

The data on which Study 1 are based are part of a larger study on narrative identity content (McLean et al., 2016; Syed \& McLean, 2016).

\section{Participants}

Participants were 245 American college students (mean age $=19.39, S D=2.80 ; 75 \%$ women; $73 \%$ White). Eighteen percent of participants had mothers and fathers with a high school degree or less, $36 \%$ of mothers and $34 \%$ of fathers had some college, $28 \%$ of mothers and $27 \%$ of fathers had a BA, and $17 \%$ of mothers and $21 \%$ of fathers had more than a BA. Data on sexual orientation, or whether participants were cisgender or transgender were not collected.

\section{Procedure}

Participants were recruited from undergraduate psychology courses, which serve a variety of students for general education requirements, as well as those who are, or intend to be, psychology majors. After providing informed consent, participants wrote narratives and completed surveys in a room alone (written with Media Lab v2008; Empirisoft, 2008). Once 
finished, participants were debriefed, thanked, and given course credit for participation, which took an average of $1.5-2$ hours.

\section{Measures}

The Ego Identity Process Questionnaire (EIPQ). The EIPQ is a 32-item survey to assess identity exploration and commitment across eight domains that are ideological (occupation, religion, politics, values) or interpersonal (friendship, dating, sex $\operatorname{roles}^{1}$, family) (e.g., "My ideas about men's and women's roles have never changed as I became older"; "I have definite views regarding the ways in which men and women should behave") (Balistreri, BuschRossnagel, \& Geisinger, 1995). We used the overall exploration and commitment scale, and the sex roles subscale (alphas range $=.71-.74$ ). Surveys assessing personality traits and well-being were also administered, but were not examined in the present study.

Narrative prompts. Participants completed eight narratives for each of the EIPQ domains, counter-balancing ideological and interpersonal, though we only examine sex role narratives. Prompts were modeled on McAdams' (2006) guided autobiography task:

Please think of an important autobiographical memory that highlights experiences with sex roles; that is, the roles that men and women should play in the world. Please take your time, and describe your memory of the event, including where you were, whom you were with, what happened, your reaction, the reaction of anyone else involved in the event, and why it is important to you.

After each narrative participants answers 18 likert items (scale ranged $1-7$ ) about the event. These questions were drawn from prior work aimed at assessing conscious representations of the relations between self and event (e.g., Pasupathi, Billitteri, Mansfield, Wainryb, Hanley \&

\footnotetext{
${ }^{1}$ We used the term sex roles, as opposed to gender roles, to be consistent with the identity status literature.
} 
Taheri, 2015). Those questions concerning the stability or change in self-views were condensed into two factors representing self-stability (e.g., "The kind of person I am explains why this experience happened the way it did." $\alpha=.80$ ) and self-change (e.g., "This experience really changed the kind of person I am - caused me to become a different type of person." $\alpha=.74$ ). Narrative Coding

The second author coded all narratives for position, specificity, and personal connection. Two research assistants, blind to study aims, completed reliability on $25 \%$ of the narratives. Meaning and valence were coded for the initial project by three research assistants, blind to study aims, who had acceptable reliability with a trained graduate student ( $10 \%$ of cases). Coders checked $20 \%$ of their codes with each other to prevent coder drift, and discussed difficult cases with the trained graduate student to reach consensus (Syed \& Nelson, 2015).

Master narrative positions. The first and second author, in consultation with the third author, conducted exhaustive reviews of all narratives to develop a coding system to identify master narrative positions. Given the prompt, and the historical weight of the 'traditional' gender narrative, this was termed the master narrative, defined by the view that men and women have separate, and traditional, roles, reflecting a clear power differential. For example, for men these roles include being breadwinners, more powerful, and stronger, and for women these roles include being submissive, nurturing, and weaker. This traditional position was the starting point of negotiation, such that individuals could align themselves with it, or use an alternative narrative, the latter of which took two forms. The first, which we termed the equality narrative, was in direct contrast to the traditional narrative, and was defined as 'same,' such that the events in the narratives were about men and women playing interchangeable roles (e.g., men and women can do the same things, or that men can be nurturing or women can be physically strong). 
The second alternative position was the 'should-be-equal' narrative. In these narratives, the event recalled was often an experience of sexism, or a story structured around the continued (unjust) influence of the traditional narrative. Reliability was acceptable for positions (Kappa $=.71,81 \%$ agreement).

Specificity. Using an established coding system (Singer \& Blagov, 2000), narratives were identified as generic, in which no specific event was disclosed (e.g., "My mom always used to cook dinner."); episodic, in which the event extended over a period longer than a day (e.g., "The summer after high school I learned how to cook."); specific, in which a specific, one-time event was reported ("The day I graduated from high school, I cooked dinner for my boyfriend.”); or as a non-narrative, in which commentary was provided but no past event, the latter of which were dropped from analyses (Kappa $=.90,93 \%$ agreement $)$.

Degree of personal connection. This code was developed for the purposes of this study to identify whose story was being told, and was only coded only on specific and episodic narratives. The range was 1 (reporter was directly involved in the event as a main protagonist), 2 (reporter was a bystander to the event), to 3 (the narrative was about someone else's experience, which the reporter did not witness) (Kappa $=.87,94 \%$ agreement).

Meaning-making. Each of the memory narratives was coded for sophistication of meaning on a four-point scale (McLean \& Pratt, 2006). A zero indicated no explanation of the meaning of the event. Narratives were scored as one if there was a specific lesson that the reporter learned from the event. A score of two was assigned to narratives that contained vague meaning; narratives of this sort describe some growth or change in the self, but the specifics of the change are not clear. Narratives were scored as three if there was evidence that the reporter gleaned specific and broad insight from the event (intraclass $r=.83-.89$ ). 
Valence. Narratives were coded for affective valence by capturing the beginning and end of the narrative as either positive, neutral, or negative (intraclass $r$ range $=.86-.92$ ).

Narratives that did not concern gender $(n=8)$ were not included, and narratives that were uncodeable for master narrative position $(n=16)$ were not included in analyses concerning positions taken ( $n=6$ overlapping between these categories).

\section{Study 1: Results and Discussion}

\section{Descriptive Statistics}

Means and percentages for all narrative variables and survey measures can be found in Table 1. The majority of the narratives were coded as employing the 'should-be-equal' position, followed by the 'equality', and 'traditional' positions. The majority were also specific memory narratives, followed by episodic, and generic. Most narratives were about events in which the reporter was directly involved, so we created a binary variable (direct versus non-direct involvement) due to these low base rates.

\section{Master Narrative Use: Demographics}

There was a marginally significant difference in narrative use by $\operatorname{sex}, \chi^{2}(2, n=243)=$ $5.73, p=.06, \mathrm{v}=.15$. Men were more likely $(\mathrm{ASR}=2.3)$ than women to use the traditional narrative. There were no significant differences in narrative use by ethnicity (White versus nonWhite), $\chi^{2}=1.85$. There were no significant differences in master narrative use by parent educational status $\left(\chi^{2}=6.21\right)$ or age $(F=1.00)$.

\section{Master Narrative Characteristics}

A single repeated measures ANOVA showed a significant main effect of narrative position on beginning and end valence, $F(2,243)=17.21, p<.001, \eta^{2}=.12$. Post-hoc tests (Tukey's test used throughout analyses) and inspection of the estimated marginal means showed 
that the equality $(m=2.14, s e=.05)$ and traditional $(m=1.96, s e=1.98)$ positions were more positive at the beginning compared to the 'should-be-equal' $(m=1.75, s e=.04)$ position. The end of the traditional $(m=2.00, s e=.08)$ and equality positions $(m=2.19, s e=.06)$ was also more positive than the 'should-be-equal' narrative position $(m=1.69, s e=.05)$. This suggests that adopting the master narrative is relatively less conflictual than engaging with alternative narratives (McLean \& Syed, 2016), or that fitting in with alternative narratives may be easier when those narratives are not in transition, or are not indicative of conflict.

There were no significant differences in narrative position for stability ratings $(F=.35)$, but there were for change ratings, $F(2,236)=7.49, p<.001, \eta^{2}=.06$. Post-hoc tests showed that traditional narratives $(m=4.06, s e=.18)$ were rated lower on change than should be $(m=4.76$, $s e=.11)$ and equality $(m=4.95, s e=.15)$ positions. For specificity, there was a marginal difference for position use, $\chi^{2}(4, n=219)=8.45, p=.08, \mathrm{v}=.08$, with fewer episodic $($ ASR $=$ 2.1) and more generic $(\mathrm{ASR}=2.1)$ in the traditional narratives, and fewer generic $(\mathrm{ASR}=-2.2)$ in the should-be-equal narratives. There were no differences in narrative position and how personal the narrative was $\left(\chi^{2}=2.70\right)$. Thus, the traditional position reflects a less changeoriented narrative, which is also rooted in less specific experiences. It is possible that the traditional narrative represents the kind of un-analyzed framework that master narratives provide those who have not had specific experiences that deviate from that framework.

\section{Identity Processes}

To our surprise there was no association between meaning-making and narrative position $(F=1.73)$, which may have been an issue of low base rates of meaning across these three types (range of means from .80 for traditional to 1.17 for should-be-equal). As expected, there was an association with position and the survey measures of identity processes. Those with an equality 
$(m=4.09, s e=.07)$ or should-be-equal $(m=4.20, s e=.05)$ positions were higher on identity exploration than those with a traditional narrative $(m=3.87, s e=.08), F(2,240)=6.30, p<.01$, $\eta^{2}=.05$. Those with traditional narratives $(m=4.07, s e=.09)$ had higher identity commitment than those with should-be-equal narratives $(m=3.85$, se $=.05), F(2,240)=3.05, p<.05, \eta^{2}=$ .03. We saw the same pattern of results within the sex roles domain for exploration, $F(2,240)=$ 5.13, $p<.01, \eta^{2}=.04$, and commitment, $F(2,240)=6.76, p<.001, \eta^{2}=.05$. These results suggest that engagement with alternative narratives is related to personal identity exploration - at least from a status perspective, and that engagement with traditional narratives reflects a conventionality in understanding the relation between self and society, perhaps akin to identity foreclosure (e.g., Berzonsky, 1989).

Building on the above interpretation, stability narratives were associated with greater overall identity commitment, $r(236)=.14, p<.05$, and greater sex role commitment, $r(236)=$ $.15, p<.05$. Those with narratives more focused on change reported more overall identity exploration, $r(236)=.14, p<.05$, and marginally more sex role exploration, $r(236)=.12, p=$ .07 . Those with direct narratives $(m=4.23, s d=.56)$ versus no direct involvement $(m=4.01, s d$ $=.57$ ), had more identity exploration, $t(161)=2.36, p<.05, d=.39$, and marginally more sex role exploration $(\operatorname{means}(s d s)=3.95(1.16)$ v. $3.61(1.09)), t(161)=1.76, p=.08, d=.31$; there were no associations with identity commitment $\left(t^{\prime} \mathrm{s}<1.19\right)$ for direct versus indirect. There were no associations between identity processes and specificity.

These results are similar to the findings for identity exploration and commitment, showing that beyond positions taken, those narratives that were more stable were more likely to be reported by those who felt more sure of themselves, and those narratives about change were more likely to reported by those in the midst of exploration. Further, when individuals identify as 
having had more direct experiences in the content domain of interest they have more to work with in terms of identity processes. In other words, when individuals either do not experience certain contents as salient, or do not attend to them, there is little material for identity exploration. It is possible, of course, that this general engagement of exploratory processes may be about identity development in general - that those who are more exploratory are willing to consider multiple, or new, aspects of any issue. We hypothesize that there may be important individual differences in cognitive complexity and the propensity for general exploration, but our recent work has also shown that not all identity contents are reflected upon, or processed, to the same degree (McLean et al., 2016).

\section{Study 2}

In Study 1 we found that more direct, or personal, experience was related to identity exploration, and that specific experiences were somewhat more common in the 'should-be-equal' position, raising the question of how personal narratives are used in negotiating with these structures. Thus, in Study 2 we had one overarching goal, which was to examine personal and master narrative positioning in vivo to capture the dynamics of how personal and master narratives interact. We were also motivated to explore interactive processes that might contribute to the rigidity and flexibility of master narratives, as interpersonal interactions are viewed as primary mechanisms of master narrative maintenance, and potentially change (e.g., Thorne \& McLean, 2003). One of the reasons that we have argued that master narratives are a part of the structure of society is that they are rigidly held and hard to change (McLean \& Syed, 2016). They are engrained in more tangible aspects of structure, such as the legal system, but they are also engrained in day-to-day discourse (e.g., Korobov \& Bamberg, 2004). Examining how individuals negotiate with them might shed light on some of the interpersonal processes that 
contribute to this rigidity. Such an examination should also tell us more about how alternative narratives are constructed in reference to the master narrative.

Consistent with discursive approaches (e.g., Korobov \& Bamberg, 2004), and with recent calls for the use of qualitative analyses in the study of emerging adulthood (Schwab \& Syed, 2015), we conducted a qualitative analysis of conversational processes. Bamberg (2004) has argued that examining interactive conversational processes is a way to understand the construction of personal identity within a "shared cultural model of sense-making" (p. 334) (see also, Korobov, 2010). That is, examining how conversation unfolds allows for an understanding of personal and master narrative construction.

For our conversational prompt we chose two newspaper articles about gender: the pay gap and biases in the hiring of research assistants. We chose these prompts because they raise questions about how the life course master narrative unfolds for men and women, and were on topics that should be of interest to this participant population given prior work on the salience of exploring adult roles at this stage. We encouraged participants to consider how their own experiences with these issues connected to larger structural issues, with time to reflect on the articles alone, to discuss them together, and then to reflect on the conversation in an interview.

\section{Study 2: Method}

\section{Participants}

Participants were 12 undergraduates drawn from the same Psychology participant pool as in Study 1, but from different years. They were strangers to each other, and paired into dyads: 2 male/male, 2 female/female, 2 male/female dyads. All participants identified as male or female (none provided an alternative), and heterosexual. All participants were White, except one who reported ethnicity as 'other,' and were between the ages of $18-24$. Five reported that their 
mothers had some college or less (five fathers), five reported that their mothers had college degrees (two fathers), and two reported that their mothers had more than post-secondary education (five fathers).

\section{Procedure}

After providing informed consent each participant was left in a room alone to read two articles. They were given materials to make notes, and the following instructions:

In this study we are interested in perceptions of news stories on a variety of topics like politics, the environment, and the economy. Your group was randomly assigned to read and discuss two articles about gender roles. Please use these highlighters and pens to note any parts you find particularly interesting. You will be having a conversation with each other after you read these, so please do read them carefully. However, you will not need to summarize the articles in the conversation, we are much more interested in your reactions to them, and any personal experiences you might have with these issues. We have included a blank piece of paper here. Once you have read the articles please take a moment to jot down any personal experiences you have had that are related, even tangentially, to the topics in the articles. That is, how does the content of these articles relate to your life? Or are they relevant to your experiences? Please let me know if you have any questions.

After 10 minutes we asked participants to talk about their perceptions of the articles and any relevant personal experiences that they had in an audio and video recorded conversation, which lasted 15 minutes. After the conversation, each participant was taken to a separate room where the play-back interview was conducted by a same-sex interviewer. The conversation was replayed for the interviewer and participant to listen to. Participants were instructed to stop the recording of the conversation at any time to comment on or explain something happening in the conversation, particularly if there was something they were thinking but did not say. Research assistants could also stop the tape if they needed clarification or had questions about what was said. This play-back, and any comments made while listening to it, was recorded as well. After the interview, participants completed several surveys, were debriefed, thanked, and given one hour of course credit. 


\section{Materials}

Participants read two articles. The first was from the New York Times (Miller, April 23, 2014), which discussed within profession differences in pay (e.g., male and female social workers) rather than between profession differences (e.g., female social worker and male engineer). The article addressed issues such as workplace flexibility in relation to caregiving as one way to alleviate the pay gap. The second article came from the New York Times' Science section (Chang, September 23, 2012), concerning a study in which male and female professors were given identical resumes for research assistant positions, with male or female applicants. Professors were more likely to hire males, regardless of professor sex, and paid females less than males if they did hire them. Participants also completed demographic surveys and three measures of gendered attitudes (gender centrality, sex role egalitarianism, attitudes towards women), which we did not examine.

\section{Analytic Process}

Our analytic framework was guided by a consensus process rather than formal reliability, given the small sample size and intention to conduct an interpretive, in-depth analysis (Syed \& Nelson, 2015). This allows for, "the discovery of experiences and subtle processes that might otherwise go unnoticed" in comparison to the analysis of larger datasets that require coding in broader strokes (Syed \& Nelson, 2015, p. 9). Critical to a consensus process is creating a diverse interpretive community, and triangulation between data and interpretation (Merriam, 2009). Consistent with this approach, the purpose was not to generalize across persons, but to identify discursive processes related to negotiating personal and master narratives.

The coding team consisted of the first and second authors and six undergraduate students who were majoring in Psychology (some of whom conducted the playback interviews), with 
varying ethnic and socio-economic backgrounds and exposure to classes and theories about gender, though the majority of the team were female. Each member of the team read each conversation and playback interview in depth and alone, and collated his or her own responses. This was followed by a holistic discussion of each case as a group. We also identified moments when personal stories were told, or lack of personal experience was referenced. As in Study 1, personal stories were defined as specific past events that happened to the reporter or someone he or she knew. Finally, we identified moments of disagreement between parties to understand where positions diverged, which was primarily evidenced by offering divergent opinions on the articles or contrasting life experiences. After the lab identified these moments in the conversation, the first author returned to the parts of the conversations and interviews that had been identified to examine processes of negotiation, in consultation with the second and third authors. We focused on these parts of the conversation because they reveal moments of 'conversational trouble' in which the dynamics of positioning are revealed (Korobov \& Bamberg, 2004). In this case, we were particularly interested in how alternative and master narratives interacted, as well as how personal experiences did or did not align with master and alternative narratives.

\section{Study 2: Results and Discussion}

\section{The Use of Personal Experience}

Personal experiences were exceedingly rare and, when used they were almost always used to refute the data, most often by reinforcing the equality narrative. More specifically, we saw resistance to the traditional narrative by using personal experience to contradict traditional roles or sexism. This was seen in all dyads. 
For example, in a male dyad one participant noted that "all the girls in my biology class are really good at it, and the guys are not. Like me." In the other male dyad, one participant refuted the data in the articles by talking about a summer job that involved physical labor.

...you would think the guys might have a bias of getting paid more, but like my boss is female and works harder than I do...she does the same work and so we both get paid the same, or she gets paid more because she's seniority...In general, like there's no, there's not a pay gap going on.

He explicitly denied the data in the articles with personal experience, and later stated that "the claim they made is super ambiguous." Similarly, in a female dyad, a participant stated that she has a friend who has researched the pay gap issue "a lot," and says, "it's actually not true."

In a male-female dyad, one participant took a slightly different tack by using personal experience to refute the data and to align herself as not feminist. She did this explicitly by talking about her friends who are feminists and would be "all over" these articles to contrast with her perspective. While she generally positioned herself within the equality narrative, at times she returned to a more traditional position. In terms of the equality narrative she pointed to her own experiences, in which she was taught that she could do anything men could - she "couldn't be lesser." She went on to say that she has always been "fiercely independent," and never even thought to consider differential treatment. During a discussion of the workplace she stated, "I wouldn't think like, 'you're gonna pay me less."' At one point she said that all of this should "bug" her more, but she still cannot engage with the articles. Returning to the traditional position she then argued that there are good reasons that women may not be hired to be CEOs or supervisors, such as their emotionality or cattiness.

A second pattern we observed was the inability to come up with personal experience in support of the data presented in the articles. In one male conversation, the participants repeatedly stated that they had no experience with the issue. They were sympathetic to the topic, but could 
not engage. They mentioned "surprise" at the data. They wondered, "why hasn't there been a change yet?" For some, this lack of experience resulted in a feeling of removal. In one male conversation, one participant said, “So, like it matters. But I don't really relate to it at all. There's nothing - I don't feel an emotional connection to it anyway," sentiments that were repeated in the follow-up interview.

We also saw an emphasis on distancing the self from the issue to not appear sexist. In one conversation, there was a good deal of discussion about generational differences. One participant stated that those who are behaving in more sexist ways are "from a completely different generation... a completely different era," and the other participant responds, "Right. Different culture." In another conversation, one woman said that the issue "does not seem that prevalent...look how far we've come!”

We wondered whether the lack of personal stories was a reflection of experience or some bias in encoding, recall, or comfort with the topic. Yet, although we were uncertain, the participants were quite sure that their inability to recall stories was due to a lack of experience. Indeed, one common theme was that the participants felt that they were not yet at the stage to notice these issues. For example, one male said, “But I'm just not, I don't know I'm not at the point in life where I'm experiencing that bias yet." A female said, "I've never put a ton of thought into it, but maybe that's because I'm not like looking for a career yet." This is inconsistent with Study 1, in which participants were able to recount personal experiences, particularly in the should-be-equal narratives, which centered on experiences that countered the equality narrative. Thus, the negotiation of personal and master narratives may be more challenging in situ, than in solitary, and also reflects their invisibility, and how they can be internalized even when explicitly stated beliefs are different. 
The only place where we saw personal experience used to explore the 'should-be-equal' narrative was in the playback interviews, always in response to interviewer questions, and all from female participants. One female recounted her observation that one of her male professors was much more informal in language and clothing than one of her female professors. She mused:

And so maybe he just has more of a sense of humor which is probably likely, but um, maybe it's also because you know she's a woman in the field, and she wants to be taken very seriously...

Another reported:

I guess like being a female athlete, cause I'm on a basketball team, (IV: Mhm) like I do see-definitely see like the men get priority above women, and like the men get bigger crowds, you know, but... There's a lot of other factors going into it and... Um, I mean... Guys can dunk and their just like more showy than girls.

It is notable that these personal experiences were shared in the interviews, and needed prompting. This suggests that some experiences may be silenced in some situations, perhaps even be invisible, unless explicitly brought to the fore.

Overall, these data suggest that personal stories are rare, unelaborated, and relatively impersonal. Many of the references centered on other's experiences (friend, sister, parents, teachers), supporting our findings from a prior study in which gender was not raised as a specific identity content in thousands of narratives, and with results of Study 1 showing that events are more specific in the should-be-equal narrative. We still do not know if people are not experiencing such events, not encoding them, or not reporting them, but it does appear that experiencing deviation from egalitarianism is rarely material for these types of conversations.

\section{Finding Common Ground and Master Narrative Rigidity}

In our analysis of moments of disagreement, we found that the most prevalent dynamic were efforts to find common ground. That is, when there were moments of disagreement, 
movement was toward each other, rather than away. This common ground then resulted in dilution of disparate positions as participants lessened their stance to meet the other half-way.

In one male-male conversation the two participants were at odds, taking classic liberal and conservative positions. The first participant refuted the data, claiming that there were no problems. The second challenged this at first, but later they agreed that things have changed, making a generational argument and moving the focus toward others. At another point they discussed socialism in terms of pay inequality, but quickly began to joke about it, and again moved to safer territory. Although the conversation moved toward more comfortable territory that appeared agreeable to both, they were clear about their divergent positions in the interviews.

In one male-female conversation, the female who called herself "fiercely independent," positioned herself as basically anti-feminist, stating that she had no experience with the issue and claiming that there is an "equal playing field." She appeared to be trying to align herself with her partner, assuming he held the same position. However, several times he puts out bids with a divergent position. At one point he asked her to reflect: "How bout you, like if you look inward, do you feel like you would have a bias towards one or the other? Or you just feel like it would be pretty equal?" He also hedged on some of her more extreme positions. However, he never fully challenged her position in the conversation, instead appearing to soften his stance as they continued talking. Yet in the interview, he expressed his own views much more clearly. At one point he commented on her joke in the conversation that he should be happy because he will be paid more, and said:

Umm. I feel like, um, I feel like a little bit with her that, uh, she, uh. How should I say this? Um, she wasn't kind of -um, not that she was missing the point or anything, I think she obviously got it, but she was thinking a lot more on a sort of anecdotal, personal, individual level versus the recognition that when you take a big enough sample you weed out just sort of picking people based on if they're strong or if they're kind, or whatever. Uh, there's still going to be bias just because of different gender and 
not uh, recognizing, not because of any other qualities, just gender and like that's the issue. And she pretty focused on just like uh, like the individual experience...

Here he raised important structural issues, at odds with her position (which she maintained in her interview), but did not disclose them explicitly in the conversation, opting for common ground. Thus, it is not only that people align themselves with one another in conversation, but also that they align themselves with where they think the other person is positioned regardless of whether this is true. In this case, her assumption about his position may have been driven by the traditional master narrative, creating somewhat of a vicious cycle.

In sum, there was little movement when there was disagreement because agreement was quickly sought out. These data are consistent with Korobov and Thorne's (2007) discussion of 'mitigation' in conversations between friends, in which tricky issues or conflict are skirted, which Korobov and Bamberg (2004) called "avoiding conversation trouble."

\section{General Discussion}

In the present study we applied the newly developed master narrative framework (McLean \& Syed, 2016) to the content domain of gender identity. We examined identity processes, attending to both personal and cultural aspects of identity development. Employing three perspectives on identity with a multi-method approach, we identified three narratives about the gendered life course: the traditional, equality, and should-be-equal narratives. Further, we found that identity processes were tied to the ways in which individuals positioned their own stories around these narratives.

In Study 1 the most common narrative was the 'should-be-equal' narrative, and use of this narrative was more likely to occur within more specific experiences. In contrast, however, the should-be-equal narrative was not commonly referenced in Study 2, and was only referenced in the play-back interview, suggesting that exploration of a more challenging alternative 
narrative may be less socially acceptable. The lack of discussion of this narrative does not only mean that it may get less air-time, but also that there is less opportunity for individuals to negotiate their personal stories with this particular alternative narrative, reflecting a lost opportunity for identity work that is enriched by sharing the self with others (e.g., Pasupathi \& Hoyt, 2009).

In terms of the intractability of master narratives we saw a push toward finding common ground. Although interpersonally more pleasant, this is a potential mechanism for the rigidity of master narratives as divergent positions are diluted when people move towards the middle. This process may be especially pronounced in social contexts because of the power inherent in master narratives, and the risk of rejection one takes in deviating (McLean \& Syed, 2016; see also Hammack, 2011). We saw this dynamic in Study 2 when the positions taken in conversation were not always the positions taken in the interview. Specifically, there was more likely to be agreement in the conversation, and expressions of disagreement in the interview.

Finally, as mentioned above, we also found a striking lack of personal stories in Study 2, despite many prompts, perhaps because experiences consistent with the should-be-equal narrative - the most common in Study 1 - were silenced. When personal stories were used, it was often to refute data by employing the equality narrative. Yet the use of the equality master narrative is one way to, ironically, maintain the traditional narrative because it suggests that there is no further work to be done. The use of personal stories is also an especially powerful way to refute data because stories are so persuasive (e.g., Green \& Brock, 2000).

Our primary intention was to apply the master narrative framework and demonstrate its viability and significance in understanding identity development. In applying it to the specific content of gender identity, there are several lessons learned that are novel to prior work on 
gender typicality and identity development more broadly. First, we argue that attention to gender as a content domain is meaningful, and this is a place where traditional identity researchers have placed far less attention compared to other domains (cf., Archer, 1989: Fivush \& Buckner, 2003), compared to domains such as ethnicity, or to religion or politics. Second, we propose that a meaningful lesson here is in providing a way to contextualize the study of gender identity (which has been largely defined as gender typicality) in individual's experiences and interactions with the culture at large, and with others. That is, gender identity is viewed here in a much broader setting that allows an understanding of the processes by which individuals learn about, challenge, and accept notions of gender into their identities, particularly as these identities related to power structures in society. We note here that there are various ways to examine gender identity, some of them are more implicit than examining specifically gendered experiences, as we did. For example, many researchers have argued that the ways in which men and women tell stories can reveal important components of their identities, such as the degree to which they elaborate on certain emotions (e.g., Fivush \& Buckner, 2003; Grysman et al., 2016). Our focus on explicit constructions of experiences related to gender is only one way to examine gender identity, but it is a way that showcases the connection between self and society.

More specifically, our results in Study 1 cohere around the idea that the traditional gender narrative is more stable, with more relations to commitment (a stable place), and less related to exploration (an unstable place); this is a narrative that is resistant to change. Further, this position may be more likely to be endorsed by males, though we were compromised by our sample size to fully test this question. This suggests that changing the norms and expectations for gender roles and the life course, may come from women and those who are interested in, able to, or forced to explore their identities. However, this change in thinking about gender - a domain so engrained 
in history, rituals, and biology - is not easy to accomplish. As we saw in Study 2, the motivation was to find common ground in social interactions, which lessened opportunities for discussing the transitional narrative that was most commonly held in Study 1, and in some of the interviews in Study 2. To put this in the language of traditional gender identity work, the maintenance of gender norms, or gendered traits, may be enforced by the lack of opportunity for discussing, or enacting, deviations from these norms, which may in turn impact the potential for changing the structures that enforce them - an unending circle.

Thus, the negotiation of the gendered master narrative appears to happen more in private, even though public awareness is critical for cultural change. This showcases the levels of impediments - from the macro structure to micro social interactions - that reinforce the gendered master narrative, limiting the possibilities for changing both cultural and personal stories, and revealing the inherent limitations of personal agency in constructing one's story (Hammack, 2011; McLean, 2015; McLean \& Syed, 2016). Although this public-private challenge may be inherent in the framework of master narratives more broadly, the historical and biological weight of gender may make this more the case here, though this is a question still to be tested. Despite these impediments to change, we also see these data representing the idea that, as much of the contemporary awareness and understanding of gender is in fluctuation, the gendered master narrative may also be changing, at least in terms of what is internalized in individual's identities.

We also note that this paper provides an example of how to integrate various approaches to identity development to more fully understand the phenomenon (McLean \& Syed, 2015; Syed \& McLean, in press). That is, each approach to identity focuses on a different angle, and combining them allows us a more multi-dimensional analysis. Further, heeding recent calls to expand methodological repertoires (Schwab \& Syed, 2015; Syed, 2015), our mixed methods 
approach allowed us a much deeper understanding of the way that gender identity development looks and develops. For example, Study 1 allowed us to quantify the types of master narratives towards which people are positioning themselves, and relate those positions to internal identity processes. Study 2 allowed us to understand more about the social dynamics of those positions, as they are enacted in everyday conversation. We imagine that this integrative methodological approach has much to offer the study of identity development.

\section{Limitations and Conclusions}

Our first limitation is the use of a college sample. Clearly these issues speak to individuals regardless of educational background and future work should examine how these processes and contents are enacted in other demographic groups. However, it is interesting that we saw a relatively high frequency of the traditional narrative $(20 \%)$, and that we saw such rigidity of master narratives. That is, in one of the more liberal, progressive groups in U.S. society (Pryor, DeAngelo, Blake, Hurtado, \& Tran, 2011), we still saw evidence of the traditional master narrative and the difficulty of changing it. It is also possible that demand characteristics were limiting what was reported, though the anonymity of Study 1, and the dual reporting contexts (conversation and interview) may have ameliorated this limitation. We also note that our data were collected in the United States. Given our emphasis on cultural master narratives, we expect that these processes may look different in cultures with different expectations and stories about the gendered life course.

We also examined one domain of identity content - gender - but we know that gender (and its multiple components) intersects in critical ways with other aspects of people, such as race, sexuality, and social class, and that these intersections may play important roles in how 
people position themselves around these narratives, as well as what narratives are available. Adding layers of complexity to this phenomenon will be important as this work builds.

We also note that our sample size in Study 1 was not ideal for detecting sex differences, and that the majority (but not all) of our coders were female, so replication of these results is important. We do note, however, that we did not hypothesize sex differences because of recent claims that it is not sex that is the critical variable, but gender identity (Grysman \& Hudson, 2013) - that is, how one positions the self in relation to these larger narratives. Of course, biological sex still retains an important role given existing power dynamics (and is reflected in our marginal results in Study 1).

Finally, it is possible that this is a developmental stage in which gender is backgrounded (Fivush \& Buckner, 2003), as other tasks take over in importance. This claim is interesting in the context of a stage where love and work become prominent concerns, and which are gendered. However, as our participants in Study 2 noted they were not quite in the stage of experiencing the push and pull between these issues, which becomes most prominent when childrearing begins (Katz-Wise, Priess, \& Hyde, 2010). Thus, other prompts might have pulled out more conversational engagement with master narratives for emerging adults.

The application of the master narrative framework to the content of gender identity has shown us how intimately connected the structural and the personal are in the processes of identity development. We see that gender identity is not something that is solely defined by one's perceptions of how gender typical one is, for example, but also by the affordances and constraints of cultural expectations. In closing, we argue that tackling the complexities of identity development necessitates integrative frameworks and methods. It is only in capturing a variety of lenses on the phenomenon that we can see more fully the contents and processes of 
identity development. And it is when we combine content and process, that we can see the intimate link between self and society. 


\section{References}

Archer, S. L. (1989). Gender differences in identity development: Issues of process, domain and timing. Journal of Adolescence, 12(2), 117-138.

Arnett, J. J. (2000). Emerging adulthood: A theory of development from the late teens through the twenties. American Psychologist, 55, 469-480.

Azmitia, M., Syed, M., and Radmacher, K. (2008). On the intersection of personal and social identities: Introduction and evidence from a longitudinal study of emerging adults. In M. Azmitia, M. Syed, \& K. Radmacher (Eds.), The intersections of personal and social identities: New Directions for Child and Adolescent Development, (pp. 120, 1-16). New York, NY: John Wiley and Sons.

Balistreri, E., Busch-Rossnagel, N. A., \& Geisinger, K. F. (1995). Development and preliminary validation of the Ego Identity Process Questionnaire. Journal of Adolescence, 18(2), 179192.

Bamberg, M. (2004). Form and functions of "slut bashing" in male identity constructions in 15-year-olds. Human Development, 47, 331-353.

Berzonsky, M. D. (1989) Identity style: Conceptualization and measurement. Journal of Adolescent Research, 4, 268-282.

Chang, K. (2012, September 23). Bias persists for women of science, study finds. The New York Times. Retrieved from http://www.nytimes.com

Crocetti, E. \& Meeus, W. (2015). The identity statuses: Strengths of a person-centered approach. In K. C. McLean \& M. Syed (Eds.), The Oxford handbook of identity development. New York: Oxford University Press.

Empirisoft. (2008) Media Lab v2008 [computer software]. http://www.empirisoft.com.

Erikson, E. H. (1968). Identity: Youth and crisis. New York: Norton. 
Fivush, R. (2010). Speaking silence: The social construction of silence in autobiographical and cultural narratives. Memory, 18, 88-98.

Fivush, R., \& Buckner, J. P. (2003). Creating gender and identity through autobiographical narratives. In R. Fivush, C. A. Haden, R. Fivush, C. A. Haden (Eds.), Autobiographical memory and the construction of a narrative self: Developmental and cultural perspectives (pp. 149-167). Mahwah, NJ: Lawrence Erlbaum.

Flanagan, C. A., Kim, T., Pykett, A., Finlay, A., Gallay, E. E., \& Pancer, M. (2014). Adolescents' theories about economic inequality: Why are some people poor while others are rich?. Developmental psychology, 50(11), 2512.

Frisén, A., \& Wängqvist, M. (2011). Emerging adults in Sweden: Identity formation in the light of love, work, and family. Journal of Adolescent Research, 26 (2), 200-221.

Green, M. C., \& Brock, T. C. (2000). The role of transportation in the persuasiveness of public narratives. Journal of personality and social psychology, 79(5), 701.

Grysman, A., \& Hudson, J. A. (2013) Gender differences in autobiographical memory: Developmental and methodological considerations. Developmental Review 33, 239-272.

Grysman, A., Merrill, N., \& Fivush, R., (2016). Emotion, gender, and gender typical identity in autobiographical memory. Memory.

Habermas, T. (2007). How to tell a life: The development of the cultural concept of biography. Journal of Cognition and Development, 8(1), 1-31.

Hammack, P. L. (2011). Narrative and the politics of identity: The cultural psychology of Israeli and Palestinian youth. New York: Oxford University Press.

Hammack, P.L., \& Cohler, B.J. (2009). Narrative engagement and stories of sexual identity: An interdisciplinary approach to the study of sexual lives. In P.L. Hammack \& B.J. Cohler (Eds.), 
The story of sexual identity: Narrative perspectives on the gay and lesbian life course (pp. 3-22). New York: Oxford University Press.

Jewell, J. A., \& Brown, C. S. (2014). Relations among gender typicality, peer relations, and mental health during early adolescence. Social Development,23(1), 137-156.

Katz-Wise, S. L., Priess, H. A., \& Hyde, J. S. (2010). Gender-Role attitudes and behavior across the transition to parenthood. Developmental Psychology, 46, 18-28.

Korobov, N. (2010). A discursive psychological approach to positioning. Qualitative research in Psychology, 7(3), 263-277.

Korobov, N., \& Bamberg, M. (2004). Positioning a "mature" self in interactive practices: How adolescent males negotiate "physical attraction" in group talk. British Journal of Developmental Psychology, 22, 471-492.

Korobov, N., \& Thorne, A. (2007). How late-adolescent friends share stories about relationships: The importance of mitigating the seriousness of romantic problems. Journal of social and personal relationships, 24(6), 971-992.

Kunnen. E. S., \& Metz, M. (2015). Commitment and exploration: the need for a developmental approach. In K. C. McLean \& M. Syed (Eds.), The Oxford handbook of identity development. New York: Oxford University Press.

Leaper, C., \& Friedman, C.K. (2007). The socialization of gender. In J. Grusec \& P. Hastings (Eds.), Handbook of socialization: Theory and research (pp. 561-587). New York: Guilford.

Martin, C. L., Ruble, D. N., \& Szkrybalo, J. (2002). Cognitive theories of early gender development. Psychological bulletin, 128(6), 903.

Mayseless O., Keren E. (2014). Finding a meaningful life as a developmental 
task in emerging adulthood: The domains of love and work across cultures. Emerging Adulthood, 2, 63-76.

McAdams, D. P. (2006). Foley Center, Guided Autobiography. Retrieved November 20, 2006, from http://www.sesp.northwestern.edu/foley/instruments/guided.

McAdams, D. P., \& McLean, K. C. (2013). Narrative identity. Current Directions in Psychological Science, 22(3), 233-238.

McLean, K. C. (2015). The Co-authored Self: Family Stories and the Construction of Personal Identity. New York: Oxford University Press.

McLean, K. C., \& Pratt, M. W. (2006). Life's little (and big) lessons: Identity statuses and meaning-making in the turning point narratives of emerging adults, Developmental Psychology, 42, 714-722.

McLean, K. C., \& Syed, M. (2015a). The Field of Identity Development Needs an Identity: An Introduction to the Handbook of Identity Development. To appear in K. C. McLean and M. Syed (Eds). The Oxford Handbook of Identity Development. New York: New York: Oxford University Press.

McLean, K. C. \& Syed, M. (2016). A Narrative Approach to Psychology: Bridging the Personal and the Cultural with the Concept of Master Narratives. Human Development.

McLean, K. C., Syed, M., and Shucard, H. (2016). Identity Statuses and Narrative Identity: Integrating Content and Process. Emerging Adulthood.

McLean, K. C. Syed, M., Yoder, A., \& Greenhoot, A.F. (2014). Identity integration: The importance of domain content in linking narrative and status approaches to emerging adult identity development. Journal of Research on Adolescence. 
McLean, K. C., \& Thorne, A. (2003). Late adolescents' self-defining memories about relationships. Developmental Psychology, 39, 635-645.

Merriam, S. B. (2009). Qualitative research: A guide to design and implementation. San Francisco, CA: Jossey-Bass.

Miller, C. (2014, April 23). Pay gap is because of gender, not jobs. The New York Times. Retrieved from http://nytimes.com

Pasupathi, M., Billitteri, J., Mansfield, C. D., Wainryb, C., Hanley, G. E., \& Taheri, K. (2015). Regulating emotion and identity by narrating harm. Journal of Research in Personality, 58, 127136.

Pasupathi, M., Brubaker, J., \& Mansour, E. (2007). Developing a life story: Constructing relations between self and experience in autobiographical narratives. Human Development, 50, 85-110.

Pasupathi, M., \& Hoyt, T. (2009). The development of narrative identity in late adolescence and emergent adulthood: The continued importance of listeners. Developmental Psychology, 45, 558-574.

Pasupathi, M., \& Wainryb, C. (2010). On telling the whole story: Facts and interpretations in autobiographical memory narratives from childhood through midadolescence. Developmental Psychology, 46, 735-746.

Pryor, J. H., DeAngelo, L., Blake, L. P., Hurtado, S. \& Tran, S. (2011). The American Freshman: National Norms Fall 2011. Higher Education Research Institute, University of California, Los Angeles.

Rubin, D. C., \& Berntsen, D. (2003). Life scripts help to maintain autobiographical memories of highly positive, but not highly negative, events. Memory \& Cognition, 31(1), 1-14.

Sayer, L. C., Bianchi, S. M., \& Robinson, J. P. (2004). Are parents investing less in children? Trends in mothers' and fathers' time with children. American journal of sociology, 110(1), 1-43. 
Schwab, J. R., \& Syed, M. (2015). Qualitative inquiry and emerging adulthood meta-theoretical and methodological issues. Emerging Adulthood, 1-12.

Shulman, S., Laursen, B., \& Dickson, D. J. (2014). Gender Differences in the Spillover Between Romantic Experiences, Work Experiences, and Individual Adjustment Across Emerging Adulthood. Emerging Adulthood, 1: 36-47.

Singer, J. A., \& Blagov, P. S. (2000). Classification system and scoring manual for self-defining autobiographical memories. Unpublished manuscript, Connecticut College.

Smith, T. E., \& Leaper, C. (2006). Self-perceived gender typicality and the peer context during adolescence. Journal of Research on Adolescence, 16(1), 91-104.

Syed, M. (2015). Theoretical and methodological contributions of narrative psychology to ethnic identity research. In C. E. Santos \& A. J. Umaña-Taylor (Eds). Studying ethnic identity: Methodological and conceptual approaches across disciplines (pp. 27-54). Washington D. C.: APA Press.

Syed, M., \& Azmitia, M. (2010). Narrative and ethnic identity exploration: A longitudinal account of emerging adults' ethnicity-related experiences. Developmental psychology, 46(1), 208-219.

Syed, M., \& McLean, K. C. (2016). Integrating the Three Levels of Personality: An Empirical Test of some Possible Structures. Manuscript in preparation.

Syed, M., \& McLean, K. C. (in press). Understanding Identity Integration: Theoretical, Methodological, and Applied Issues. Journal of Adolescence.

Syed, M., \& Nelson, S. C. (2015). Guidelines for Establishing Reliability When Coding Narrative Data. Emerging Adulthood, 3, 375-387.

Thorne, A. (2004). Putting the person into social identity. Human Development, 253, 1-5. 
Thorne, A., \& McLean, K. C. (2003). Telling traumatic events in adolescence: A study of master narrative positioning. In R. Fivush \& C. Haden (Eds.), Connecting culture and memory: The development of an autobiographical self (pp. 169-186). Mahwah, NJ: Erlbaum.

Thorne, A., \& Nam, V. (2007). The life story as a community project. Human Development, 50, $119-123$.

Umaña-Taylor, A. J., Quintana, S. M., Lee, R. M., Cross, W. E., Rivas-Drake, D., Schwartz, S. J., Syed, M., Yip, T., Seaton, E., \& Ethnic/Racial Identity Study Group. (2014). Ethnic and racial identity revisited: An integrated conceptualization. Child Development, 85(1), 21-39.

Way, N., \& Rogers, O. (2015). “[T]hey say Black men won’t make it, but I know I'm gonna make it": Ethnic and racial identity development in the context of cultural stereotypes. To appear in K. C. McLean and M. Syed (Eds). The Oxford handbook of identity development. New York: Oxford University Press.

Wood, W., \& Eagly, A. H. (2015). Two traditions of research on gender identity. Sex Roles, 1-13.

Zaman, W. \& Fivush, R. (2011). Intergenerational narratives and adolescents' emotional wellbeing, Journal of Adolescence, 21, 703-716. 
Table 1.

Descriptive Statistics for Study 1 Variables

\begin{tabular}{|c|c|c|}
\hline & Mean (SD) & $\underline{\text { Percent }}$ \\
\hline Meaning & $1.11(1.35)$ & \\
\hline Beginning Valence & $1.92(.50)$ & \\
\hline End Valence & $1.89(.58)$ & \\
\hline Identity Exploration & $4.10(.57)$ & \\
\hline Sex Roles & $3.69(1.20)$ & \\
\hline Identity Commitment & $3.95(.61)$ & \\
\hline Sex Roles & $3.12(1.14)$ & \\
\hline \multicolumn{3}{|l|}{ Master Narrative Position } \\
\hline Traditional & & $21 \%$ \\
\hline Equality & & $27 \%$ \\
\hline Should-be-equal & & $52 \%$ \\
\hline \multicolumn{3}{|l|}{ Episodic } \\
\hline Generic & & $22 \%$ \\
\hline Episodic & & $25 \%$ \\
\hline Specific & & $42 \%$ \\
\hline Other & & $11 \%$ \\
\hline \multicolumn{3}{|l|}{ Personal Connection } \\
\hline Direct & & $70 \%$ \\
\hline Bystander & & $25 \%$ \\
\hline Not Present & & $5 \%$ \\
\hline
\end{tabular}

